\title{
Physicochemical characterization by AFM, FT-IR and DSC and biological assays of a promising antileishmania delivery system loaded with a natural Brazilian product ${ }^{\text {is }}$
}

\author{
Franciane Marquele-Oliveira ${ }^{\mathrm{a}, *}$, Elina Cassia Torres ${ }^{\mathrm{a}}$, Hernane da Silva Barud ${ }^{\mathrm{b}, \mathrm{c}}$, \\ Karina Furlani Zoccal ${ }^{\mathrm{d}}$, Lúcia Helena Faccioli ${ }^{\mathrm{d}}$, Juliana I. Hori ${ }^{\mathrm{e}}$, \\ Andresa Aparecida Berretta ${ }^{\mathrm{a}, \mathrm{f}, *}$ \\ a Laboratório de Pesquisa, Desenvolvimento e Inovação, Apis Flora Indl. Coml. Ltda., Ribeirão Preto, SP, Brazil \\ b Instituto de Química, Universidade Estadual Paulista, UNESP, Caixa Postal 355, 14800-900, Araraquara, SP, Brazil \\ ' Grupo de Química Medicinal e Medicina Regenerativa (QUIMMERA)-Centro Universitário de Araraquara/UNIARA, Araraquara, SP, Brazil \\ d Departamento de Análises Clínicas, Toxicológicas e Bromatológicas. Faculdade de Ciências Farmacêuticas de Ribeirão Preto, Universidade de São Paulo, \\ Brazil \\ e Departamento de Biologia Celular, Molecular e Bioagentes Patogênicos, Universidade de Sa ̃ Paulo, Faculdade de Medicina de Ribeira o Preto, \\ FMRP/USP, 14049-900, Ribeira o Preto, SP, Brazil \\ ${ }^{\mathrm{f}}$ Departamento de Ciências Farmacêuticas, Faculdade de Ciências Farmacêuticas de Ribeirão Preto, FCFRP/USP, 14049-900, Ribeirão Preto, SP, Brazil
}

\section{A R T I C L E I N F O}

\section{Article history:}

Received 16 October 2015

Received in revised form 16 January 2016

Accepted 19 January 2016

Available online 28 January 2016

\section{Keywords:}

Solid lipid nanoparticle (SLN)

Anti-leishmanial activity

Sustained drug release

Ocotea duckei

Macrophage cytotoxicity

\begin{abstract}
A B S T R A C T
The control and treatment of Leishmaniasis, a neglected and infectious disease affecting approximately 12 million people worldwide, are challenging. Leishmania parasites multiply intracellularly within macrophages located in deep skin and in visceral tissues, and the currently employed treatments for this disease are subject to significant drawbacks, such as resistance and toxicity. Thus, the search for new Leishmaniasis treatments is compulsory, and Ocotea duckei Vattimo, a plant-derived product from the biodiverse Brazilian flora, may be a promising new treatment for this disease. In this regard, the aim of this work was to develop and characterize a delivery system based on solid lipid nanoparticles (SLN) that contain the liposoluble lignan fraction (LF) of Ocotea duckei Vattimo, which targets the Leishmania phagolysosome of infected macrophages. LF-loaded SLNs were obtained via the hot microemulsion method, and their physical and chemical properties were comprehensively assessed using PCS, AFM, SEM, FT-IR, DSC, HPLC, kinetic drug release studies, and biological assays. The size of the developed delivery system was $218.85 \pm 14.2 \mathrm{~nm}$, its zeta potential was $-30 \mathrm{mV}$ and its entrapment efficiency (EE\%) was high (the EEs\% of YAN [yangambin] and EPI-YAN [epi-yangambin] markers were $94.21 \pm 0.40 \%$ and $94.20 \pm 0.00 \%$, respectively). Microscopy, FT-IR and DSC assays confirmed that the delivery system was nanosized and indicated a core-shell encapsulation model, which corroborated the measured kinetics of drug release. The total in vitro release rates of YAN and EPI-YAN in buffer (with sink conditions attained) were $29.6 \pm 8.3 \%$ and $34.3 \pm 8.9 \%$, respectively, via diffusion through the cellulose acetate membrane of the SLN over a period of $4 \mathrm{~h}$. After $24 \mathrm{~h}$, the release rates of both markers reached approximately $45 \%$, suggesting a sustained pattern of release. Mathematical modeling indicated that both markers, YAN and EPI-YAN, followed matrix diffusion-based release kinetics (Higuchi's model) with an estimated diffusion coefficient $(D)$ of $1.3 .10^{-6} \mathrm{~cm}^{2} / \mathrm{s}$. The LF-loaded SLNs were non-toxic to murine macrophages (20-80 $\mu \mathrm{g} \mathrm{mL}^{-1}$ range) and exerted a prominent anti-leishmanial effect $\left(20 \mu \mathrm{g} \mathrm{mL}^{-1}\right)$. These data suggest this new and well-characterized lipid nanoparticle delivery system safely and effectively kills Leishmania and warrants further clinical investigation.
\end{abstract}

(c) 2016 Published by Elsevier B.V.
放 Selected paper from 26th International Symposium on Pharmaceutical and Biomedical Analysis (PBA 2015), July 5th to 8th, 2015, Tbilisi, Georgia.

* Corresponding authors at: Triunfo street, 945, Ribeirão Preto/SP, CEP 14020-670, Brazil.

E-mail addresses: fmarqueleoliveira@gmail.com (F. Marquele-Oliveira), andresaberretta@hotmail.com (A.A. Berretta). http://dx.doi.org/10.1016/j.jpba.2016.01.045 0731-7085/@ 2016 Published by Elsevier B.V.

\section{Introduction}

Drug delivery systems based on lipid nanoparticles constitute an area of extensive development due to the prominent advantages of this colloidal system as a therapeutic. In general, solid 
lipid nanoparticles (SLN) and nanostructured lipid carriers (NLC) are highly stable and nontoxic. They can encapsulate both lipophilic and hydrophilic drugs and have been administered via several routes [1]. Moreover, they permit controlled and sustained drug release [2] and can be employed for organelle-specific disease treatments [3].

Studies of SLNs loaded with lipophilic drugs have yielded promising results. For example, the antibacterial responses of norfloxacin were enhanced when the drug was loaded into SLNs [4]. Moreover, Bose and Michniak observed that SLN encapsulation increased the gastrointestinal absorption of quercetin, as indicated by an increase in the drug bioavailability of 571.4\% [5]. Cytotoxic compounds, such as topotecan and doxorubicin, have also been loaded into SLNs and have gained broad acceptance in this formulation as cancer treatment due to increases in drug efficacy and decreases in toxicological effects [6,7]. Notably, most lipids used to manufacture these particles are GRAS (Generally Recognized as Safe) and have low toxicity concerns [8].

Leishmaniasis, one of the main neglected and infectious diseases worldwide, is caused by parasites of the genus Leishmania, which multiply within macrophages to cause cutaneous and visceral pathologies. Leishmaniasis affects approximately 12 million people in 88 countries and its control is an important challenge for the scientific community, government and industry initiatives [9]. Pentavalent antimonials, the antimoniate of $\mathrm{N}$-methyl-glucamine (Glucantime $^{\circledR}$ ) and sodium stibogluconate (Pentostan ${ }^{\circledR}$ ) [9] followed by pentamidine and amphotericin $B$ are currently the main strategies for leishmaniasis treatment. However, these drugs are intravenously administered for prolonged periods (at least 20 days), and they are associated with off-target toxicity and occasional low effectiveness, which may lead to treatment failure.

In the search for new anti-leishmania products, Ocotea duckei Vattimo, a plant originating from Brazilian biodiversity, is considered a promising lignan-based drug that targets Leishmaniasis. A purified fraction (lignan Fraction-LF) from Ocotea duckei Vattimo is highly lipophilic and rich in yangambin lignan (YAN) and its epimer, epi-yangambin (EPI-YAN), which have demonstrated promising activity against Leishmania amazonensis and Leishmania chagasi. Monte-Neto and collaborators showed that LF inhibited the growth of the parasite in vitro at a higher rate than the current reference drug, Glucantime ${ }^{\circledR}[10]$. Significant alterations in cellular morphology and physiology associated with apoptotic cell death and autophagic characteristics have been observed in yangambintreated parasites [11]. In addition, this lignan has demonstrated low cytotoxicity in macrophages, with $\mathrm{CC}_{50}$ values of $246.7 \mu \mathrm{g} \mathrm{mL}-1$ in an MTT assay, and low antimitotic and teratogenic potential at doses not exceeding $500 \mu \mathrm{g} \mathrm{mL}^{-1}$ [12].

To administer the drug to an intracellular target, this work proposes loading SLNs with LF to safely treat cutaneous and visceral leishmaniasis. In addition to permitting administration via several routes, LF-loaded SLN may also reach the Leishmania phagolysosome [13] of infected macrophages in deep tissues. Data from the literature indicate that SLN can penetrate cell membranes [14], which permits the sustained intracellular release of LF.

A report focusing on LF composition, main compounds achievement and characterization (YAN and EPI-YAN) has recently been released. A RP-HPLC-DAD method was developed and validated: specifically, the identity and purity of markers were assessed based on their UV profile, IR, ESI-IT MS, ${ }^{13} \mathrm{C}$ NMR, ${ }^{1} \mathrm{H}$ NMR and the melting point range. Additionally, preliminary cytotoxicity analyses of murine macrophages demonstrated the safety of LF, but not of a LF-loaded nanoparticle formulation [15]. As a consequence, the present work aimed to develop a new and safe LF carrier system, as well as, to fully characterize it by PCS, AFM, SEM, FT-IR, DSC, HPLC and kinetic drug release studies. The present carrier system is supposed to target not only the pathophysiology of leishmania- sis but also to examine the properties of LF to improve the in vivo distribution and absorption of LF. Therefore, to this end, this work also presents for the first time the in vitro safety and efficacy protocols to highlight the potential medicinal uses of this new approach for Leishmaniasis treatment. The development of a safe and nontoxic lignan-based nanomedicine may provide numerous benefits for patients suffering from the side effects of the current treatments for Leishmaniasis.

\section{Materials and methods}

\subsection{Material and reagents}

The lignan fraction and the isolated pure yangambin (YAN) and epi-yangambin (EPI-YAN) compounds were obtained from the dried leaves and stem bark of Ocotea duckei Vattimo (Lauraceae) according to a previously described methodology $[10,15]$. The plant material was collected in February of 2010 in the state of Paraíba (Northeast from Brazil) and identified by Prof. Dr. Maria de Fátima Agra (Cbiotec/UFPB). A voucher specimen (AGRA 4309) was deposited in the Lauro Pires Xavier herbarium at the Federal University of Paraíba (UFPB). The experimental data from the isolated compounds confirmed the YAN and EPI-YAN identity as previously described [15].

The acetonitrile and methanol used herein were of HPLC grade (JT Baker, Philipsburg, NJ, USA). Acetic acid, dichloromethane and ethanol were of analytical grade (Vetec, Duque de Caxias, RJ, Brazil). Reagent-grade methanol was used to isolate yangambin (YAN) and epi-yangambin (EPI-YAN) and obtained from Merck (Darmstadt, Germany). Ultrapure water (Type I) was used for HPLC and obtained using a Milli-Q water purification system (Millipore, Bedford, MA, USA).

\subsection{Lipid carrier preparation}

SLNs were obtained via the microemulsion method as previously described $[6,7]$ with minor modifications. Specifically, the SLNs were prepared using stearic acid (Sigma-Aldrich, St. Louis, MO, USA) as the internal phase, soy lecithin (Lipoid GMBH, Ludwigshafen, German) as the surfactant, sodium taurodeoxycholate (Sigma-Aldrich, St. Louis, MO, USA) as a co-surfactant and purified water as the continuous phase. The microemulsion for the SLNs was prepared as follows: stearic acid (20\%) was first melted at $10^{\circ} \mathrm{C}$ above its melting point $\left(65.0-70.0^{\circ} \mathrm{C}\right)$, surfactant $(10 \%)$ was then added and stirred until it was completely dissolved. The aqueous phase (sufficient to prepare $1 \mathrm{~g}$ of microemulsion) containing the co-surfactant $(2.5 \%)$ was then heated to $80^{\circ} \mathrm{C}$ and added to the melted lipid mixture. The mixture was maintained stirring at $80^{\circ} \mathrm{C}$ until the microemulsion formed. Subsequently, $2 \% \mathrm{LF}$ was added to the resulting microemulsion, which was dispersed in purified water at $2-5{ }^{\circ} \mathrm{C}$ under mechanical stirring $(20,000 \mathrm{rpm}$ for $10 \mathrm{~min}$, Ultra Turrax, AT15, IKA-Werke, Staufen, Germany). The LF-unloaded SLNs were also prepared in the absence of the lignan extract. In some cases, as described below, the SLNs were lyophilized using sucrose as cryoprotector (7.5\%). The lyophilized SLNs were readily resuspended in ultrapure water when necessary.

\subsection{Photon correlation spectroscopy and zeta potential}

The average diameter and polydispersivity index (PdI) of the SLNs were measured by photon correlation spectroscopy (PCS) (Malvern Zetasizer Nano ZS90, Malvern instruments Ltd., Worcestershire UK) with a $50 \mathrm{mV}$ laser. The SLN suspensions were diluted $1000 \times$ in purified water before analysis. The measurements were performed at $25^{\circ} \mathrm{C}$ at a fixed angle of $173^{\circ}$. The SLN zeta potential 
was measured using the same equipment. Prior to the measurements, the SLN samples were diluted with 1:10 in $\mathrm{NaCl}(10 \%)$ solution. Each sample was analyzed in triplicate.

\subsection{SLN morphology-scanning electron microscopy (SEM) and atomic force microscopy (ATM)}

The SLN morphology was viewed using a field emission gun scanning electron microscope (FEG-SEM) (FEG XL30, Philips, Amsterdam, The Netherlands) at an accelerating voltage of $20 \mathrm{kV}$. One drop of the nanoparticle suspension was placed on a graphite surface. After drying at room temperature, the sample was coated with gold using ion sputtering. To evaluate the morphology and confirm the size of the nanoparticles, the samples were also analyzed by atomic force microscopy (AFM). To this end, samples were placed on a plate of mica and dried at room temperature. The samples were then examined on a model 9600-SPM atomic force microscope (Scanning Probe Microscopy-Shimadzu, Kyoto, Japan).

\subsection{FT-IR}

FT-IR spectra were obtained with a PerkinElmer spectrometer, model 2000 (Waltham, MA, USA). The samples were milled, mixed with dried $\mathrm{KBr}$ in known proportions and pressed into pellets.

\subsection{DSC}

DSC assays were carried out using an SDT 2960 instrument from TA Instruments (New Castle, DE, USA). The samples were heated from $25^{\circ} \mathrm{C}$ to $450^{\circ} \mathrm{C}$ at a constant rate of $10^{\circ} \mathrm{C} \mathrm{min}^{-1}$ under a nitrogen flow of $70 \mathrm{~mL} \mathrm{~min}^{-1}$.

\subsection{HPLC analysis}

YAN and EPI-YAN were assessed as previously described [15]. Briefly, the analyses were conducted using a Shimadzu (Kyoto, Japan) liquid chromatograph equipped with a CBM-20A controller, a LC-20AT quaternary pump, a SPD-M 20A diode-array detector, and the Shimadzu LC solution software, version 1.21 SP1. A Shimadzu Shim-Pack CLC-ODS column $(4.6 \mathrm{~mm} \times 250 \mathrm{~mm}$, particle diameter of $5 \mu \mathrm{m}$, pore diameter of $100 \AA$ ) was used. The mobile phase consisted of 50:50 acetonitrile:water at a flow rate of $1 \mathrm{~mL} \mathrm{~min}-1$. The injection volume was $20 \mu \mathrm{l}$. The column oven was set to $30^{\circ} \mathrm{C}$, and detection was set to $215 \mathrm{~nm}$.

The standard solutions were prepared in acetonitrile by dissolving the appropriate amount of YAN and EPI-YAN, which were previously isolated by semi-preparative HPLC-DAD, using an ultrasonic bath ( $2 \mathrm{~min}$ ) and vortex agitation. Mixtures of $100 \mu \mathrm{g} \mathrm{mL}^{-1}$ YAN and EPI-YAN were prepared from stock solutions and used to prepare working solutions. Working solutions were prepared daily in acetonitrile:water (50:50) and ranged in concentration from $0.5-20 \mu \mathrm{g} \mathrm{mL}^{-1}$. For further details and method validation, please, see reference [15]. Additional, validation parameters were also studied, in this case, for the epimers determination in SLNs, e.g. selectivity and recovery.

\subsection{Entrapment efficiency, drug loading and drug recovery}

YAN and EPI-YAN were quantified by HPLC as previously described. The entrapment efficiency (EE), which corresponded to the percentage of YAN and EPI-YAN encapsulated within and adsorbed on the nanoparticles, was determined via Eq. (1) [7]. Specifically, $1 \mathrm{~mL}$ of SLN dispersion was transferred to the upper chamber of ultrafilter (NWCO100KD) in an AMICON ultracentrifuge tube (Millipore, Bedford, MA, USA), and the tubes were centrifuged at $3200 \times g$ (Eppendorf centrifuge, 5810R, Hamburg, Germany) for
20 min. The amounts of free YAN and EPI-YAN were then measured in the filtrate. The total amount of drug in the aqueous dispersion was obtained by solubilizing an SLN dispersion aliquot in methanol and then diluting it with purified water, filtering, and injecting it into the HPLC apparatus. The entrapment efficiency (EE), drug loading (DL) and drug recovery (DR) were determined using the following equations:

$\mathrm{EE}(\% \mathrm{w} / \mathrm{w})=\left[\frac{M_{\text {total drug_}} M_{\text {free drug }}}{M_{\text {totaldrug }}}\right]$

where " $M_{\text {totaldrug" is the mass of total drug detected in the total }}$ aqueous dispersion and the " $M_{\text {freedrug }}$ " is the mass of free drug detected in the filtrate of the aqueous dispersion.

$\mathrm{DL}(\% \mathrm{w} / \mathrm{w})=\left[\frac{M_{\text {total drug }}-M_{\text {free drug }}}{M_{\text {lipids }}}\right] \times 100$

where " $M_{\text {lipid }}$ " is the mass of total lipid in the formulation containing the lignan fraction.

$\mathrm{DR}(\% \mathrm{w} / \mathrm{w})=\left[\frac{M_{\text {total drug }} \times 100}{M_{\text {added drug }}}\right]$

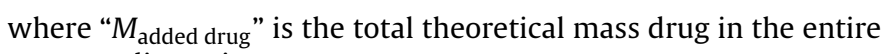
aqueous dispersion.

\subsection{In vitro kinetics of YAN and EPI-YAN release from $L F$-loaded SLN}

In vitro release studies were performed using a modified Franz diffusion cell at $37^{\circ} \mathrm{C}$. A cellulose acetate membrane (MWCO 12,000-14,000, Fisher Scientific, Hampton, NH, USA) was used as a support for the formulations. First, $250 \mu \mathrm{L}$ of the SLN suspension at a concentration of $484.3 \mu \mathrm{g} \mathrm{mL}^{-1}$ of YAN and $591.5 \mu \mathrm{g} \mathrm{mL}^{-1}$ of EPI-YAN was placed in the donor compartment (sink conditions considered), and the receiving compartment $(10 \mathrm{~mL})$ was filled with phosphate buffer $\left(10 \mathrm{mmol} \mathrm{L}^{-1}\right.$, containing $133 \mathrm{mmol} \mathrm{L}^{-1}$ $\mathrm{NaCl}$ at $\mathrm{pH}$ 7.4, from Merck, Darmstadt, Germany) containing PEG-40 hydrogenated castor oil (6\%, from Croda, East Yorkshire, UK). Samples from the receiving solution $(500 \mu \mathrm{L})$ were collected from the Franz cells at predetermined intervals and replaced with fresh receiving solution. The samples were analyzed by HPLC as described above. Mathematical dilution adjustments were further considered to correctly determine the marker contents in the samples. The release profile of the formulations (LF-loaded SLNs and solution) was evaluated mathematically following the models of zero-order (concentration versus time), pseudo first order (Higuchi's model, concentration versus square root of time) and first order (Log concentration versus time). The correlation coefficients $(r)$ of the linear fraction of the release curves were established through the slope of the line obtained by linear regression of the release curve constructed following the different release models. LF-unloaded SLNs were similarly treated and used as blank for the measurements. The experiments were carried out five times. LF solution was also assessed by solubilizing it in propyleneglycol at a concentration similar to the SLNs.

\subsection{Bone marrow-derived macrophages (BMDMs) preparation}

Bone marrow-derived macrophages (BMDMs) from C57BL/6 mice were prepared as previously described [16]. Briefly, bone marrow cells from the femurs of adult mice were cultured for 7 days in RPMI 1640 containing 20\% fetal bovine serum (FBS) and 30\% L-929 cell conditioned media (LCCM). The macrophages were replated one day prior the treatment and maintained at $37^{\circ} \mathrm{C}$ in an atmosphere of $5 \% \mathrm{CO}_{2}$ in RPMI 1640 media containing 10\% FBS and 
5\% LCCM. Cell culture medium and FBS were provided by Gibco, Invitrogen, (Carlsbada, CA, USA).

\subsection{Cytotoxicity assay}

The toxicity LF-loaded and unloaded nanoparticles was assessed based on the pore formation in the cell plasma membrane of mammalian macrophages. The pore formation was measured by quantifying propidium iodide uptake via fluorescence measurements. To this end, BMDMs $\left(1 \times 10^{5}\right)$ were seeded in black/white 96-well plates (Corning, NY, USA) and treated with LF, LF-loaded nanoparticles and unloaded nanoparticles at various concentrations for $18 \mathrm{~h}$. The treatments were carried out in phenol-red free RPMI 1640 media containing $20 \mathrm{mM}$ HEPES and $6 \mu \mathrm{g} \mathrm{mL}^{-1}$ propidium iodide (Sigma-Aldrich, St. Louis, MO, USA). Legionella pneumophila was employed as positive control because this bacterium is known to produce pores in the macrophage cell membrane after a few hours of infection [17]. The plates were incubated at $37^{\circ} \mathrm{C}$ in a FlexStation3 Microplate Reader (Molecular Devices, Sunnyvale, CA, USA), and the propidium iodide fluorescence was measured every $10 \mathrm{~min}$.

\subsection{Leishmanicidal activity}

The inhibition of cell growth was assayed in 96-well plates by cultivating promastigote Leishmania amazonensis $(1 \times 105)$ in $200 \mu \mathrm{L}$ of RPMI medium in the absence of phenol red (Sigma-Aldrich, St. Louis, MO, USA) with LF and LF-loaded nanoparticles at a concentration of $20 \mu \mathrm{g} \mathrm{mL}^{-1}$. DMSO was employed as a positive control. After $24 \mathrm{~h}, 10 \mu \mathrm{L}$ of $3-(4,5-$ dimethylthiazol-2-yl)-2,5-diphenyltetrazolium bromide (MTT) was added (Sigma-Aldrich, St. Louis, MO, USA) [18], and the cells were incubated for $4 \mathrm{~h}$. Subsequently, $50 \mu \mathrm{L}$ of $20 \%$ sodium dodecyl sulfate (SDS, Sigma-Aldrich, St. Louis, MO, USA) in $0.01 \mathrm{M} \mathrm{HCl}$ was added to each well, and the plate was incubated at room temperature until all precipitates had dissolved. The absorbance was then measured at $570 \mathrm{~nm}$ with a spectrophotometer (mQuanti, Bio-Tek Instruments, Inc., Winooski, VT, USA) and was directly proportional to the cell viability. The stimuli are expressed as the percentage of the observed cytotoxicity compared with the unstimulated control Leishmania parasite.

\section{Results and discussion}

\subsection{Photon correlation spectroscopy and zeta potential}

The average diameter of the LF-loaded SLNs was on the order of nanometers (Table 1 ), which corroborated the findings of other studies that examined similar SLN formulation and production process [9]. Moreover, the polydispersity index (approximately $0.4-0.5$ ) suggest that the suspension may require high-pressure homogenization to decrease its distribution. This type of procedure has been shown to improve this parameter as already demonstrated for a similar lipid nanocarrier [19]. Nevertheless, our results were promising despite the unavailability of a high-pressure homogenizer.

A zeta potential analysis representing the SLN electric potential at the plane of shear is demonstrated in Table 1. Zeta potential values higher than [30] $\mathrm{mV}$ are stable and exhibit a long shelf life, indicating that these colloidal systems are physically stable during storage [7]. In the present study, the zeta potential of the LF-loaded SLN suspensions was approximately $-30 \mathrm{mV}$ both before and after lyophilization.

\subsection{SLN morphology-SEM and AFM analysis}

Fig. 1(A and B) show the SEM images of the LF-loaded SLNs, which were similar to those of the unloaded lipid delivery system. As demonstrated in literature [7], particles were anisometric in shape and approximately $200-300 \mathrm{~nm}$ in size. Moreover, the particles generally agglomerated due to the lipid nature of carriers, the presence of the surfactant and the sample preparation prior to SEM analysis (Fig. 1A). Spherical and non-spherical shapes [20] of SLN have also been observed by transmission electron microscopy (TEM). Fig. 1C shows the atomic force microscopy images of the particle surface and confirms the nanometric size of both the lyophilized and non-lyophilized particles, as corroborated by data in Table 1. Additionally, no roughness is observed on the particle surface.

\subsection{FT-IR}

FT-IR spectroscopy was used to investigate possible interactions between solid-state pre-cursors of the solid lipid nanoparticles. Fig. 2 shows the FT-IR spectra of the stearic acid, soy lecithin, LFloaded SLN, unloaded SLN and LF.

Specifically, it was observed that stearic acid consists of two important sections, a carboxylic head and hydrocarbon tail (Fig. 2A). The signal at $1701 \mathrm{~cm}^{-1}$ was due to the absorption of the $\mathrm{C}=\mathrm{O}$ bond stretching in the carboxylic group $(\mathrm{COOH})$. Other peaks included hydrogen-bonded $\mathrm{O}-\mathrm{H}$ stretching at $3500 \mathrm{~cm}^{-1}, \mathrm{C}-\mathrm{H}$ stretching at $2918 \mathrm{~cm}^{-1}$ and $1424 \mathrm{~cm}^{-1}$, and $\mathrm{C}-\mathrm{H}$ bending at $1500 \mathrm{~cm}^{-1}[21]$.

The FT-IR spectrum of lecithin shows peaks at $3710 \mathrm{~cm}^{-1}$ and $3400 \mathrm{~cm}^{-1}$, which indicates the presence of $\mathrm{N}-\mathrm{H}$ stretching and $\mathrm{O}-\mathrm{H}$ stretching of amino group and hydroxyl group, respectively (Fig. 2B). Another band at $2960-2860 \mathrm{~cm}^{-1}$ indicates the presence of $\mathrm{C}-\mathrm{H}$ group of alkane. The band at $1745 \mathrm{~cm}^{-1}(\mathrm{C}=\mathrm{O})$ indicates the ester linkage. Another band at $1640 \mathrm{~cm}^{-1}$ was attributed to aliphatic alkanes. Finally, the band at $1245 \mathrm{~cm}^{-1}$ was attributed to a $\mathrm{P}=\mathrm{O}$ (phosphomoyl) group [22].

Overall, the spectra of the unloaded SLN in Fig. 2C can be considered the sum of the stearic acid and lecithin spectra. Strong chemical interactions between the precursors were not evident. These results indicate that the chemical structure of stearic acid and lecithin did not change, i.e., the initial structures are preserved on the nanoparticle preparation [21].

As expected, the spectrum of the LF-loaded SLN sample was very similar to that of the unloaded SLN. As illustrated in Fig. 2D, the main peaks were at $3500 \mathrm{~cm}^{-1}$ (hydrogen-bonded O-H stretching) and $2919 \mathrm{~cm}^{-1}$ (aliphatic C-H stretching). The peak at $1700 \mathrm{~cm}^{-1}$ observed in the spectra of pure stearic acid, lecithin and extract was almost absent in the SLN spectrum and had slightly shifted to $1761 \mathrm{~cm}^{-1}$. These studies corroborated that LF and SLN did not significantly interact.

The FT-IR spectra also suggest that LF is well dispersed in the SLN particles. Specifically, LF bands could not be clearly identified in the nanoparticle spectra due to the small amount of drug in the system and because a part of the spectrum may be covered by the bands of the precursors localized in the same region.

\subsection{DSC}

Solid lipid nanoparticles (SLNs) were produced by cooling the hot microemulsion from 75 to $5^{\circ} \mathrm{C}$ because this temperature is below the crystallization temperature of the mixed lipid phase. DSC confirmed that this process effectively produced nanoparticles. The sample was cooled in the DSC to monitor crystallization (Fig. 3).

The DSC curve of stearic acid (Fig. 3A) shows an endothermic peak attributed to melting point detected at $71.82^{\circ} \mathrm{C}$ and corresponding crystallization peak at $52.16^{\circ} \mathrm{C}$ during the second run. 
Table 1-

Average diameter, polidispersivity index (PdI) and zeta potential obtained for LF-loaded SLN lyophilized or not $(n=3)$.

\begin{tabular}{|c|c|c|c|}
\hline Nanoparticle & Average diameter (nm) & PdI & Zeta potential $(\mathrm{mV})$ \\
\hline LF-loaded SLN, not lyophilized & $218.8 \pm 14.2$ & $0.44 \pm 0.4$ & $-30.0 \pm 0.0$ \\
\hline LF-loaded SLN, lyophilized & $288.1 \pm 18.9$ & $0.56 \pm 0.1$ & $-29.0 \pm 0.0$ \\
\hline
\end{tabular}
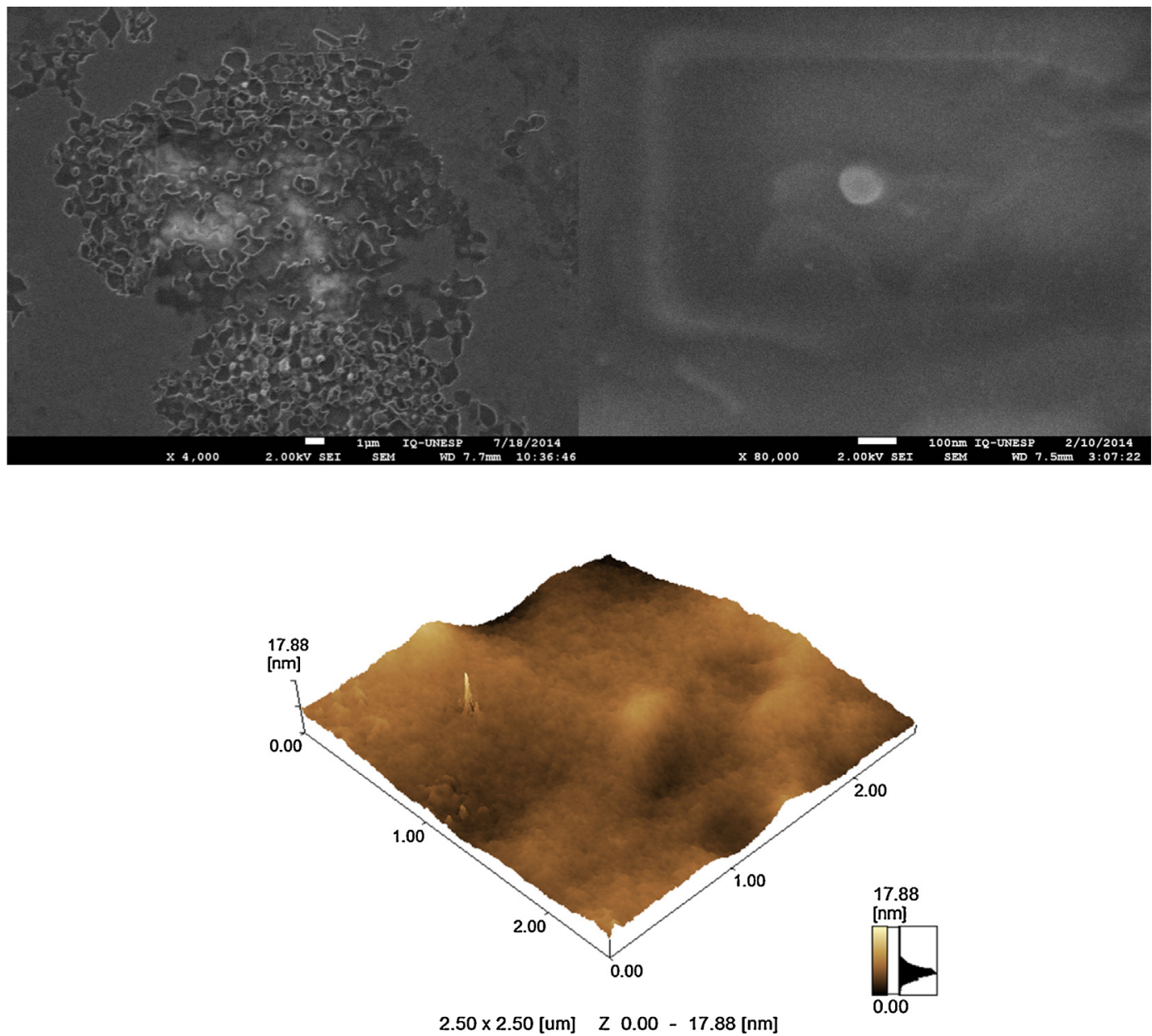

Fig. 1. SEM and ATM micrographs of LF-loaded SLN. (A) and (B) SEM micrographs (C) AFM micrograph (2 mm x $2 \mathrm{~mm}$, three-dimensional image).

Based on a comparison with the DSC curve of lecithin, it was observed a mixture of complex endotherm peaks between $150^{\circ} \mathrm{C}$ and $300^{\circ} \mathrm{C}$, which was likely due to the melting point and degradation processes (Fig. 3B).

The LF extract consisted primarily of organic components, i.e., yangambin and epi-yangambin, as observed by HPLC. The DSC curve revealed a mixture of complex endotherm peaks between ambient temperature $\left(25^{\circ} \mathrm{C}\right)$ and $350^{\circ} \mathrm{C}$ (Fig. 3C). These endothermic peaks attributed to volatile solvent evaporation and the different degradation processes characteristic to the nature of organic compounds.

DSC curves for unloaded SLN and LF-loaded SNL are shown in Fig. 3D and E. Concerning to LF-loaded SLN curve, the profile was similar to the unloaded SLN curve and it is also suggested that incorporation of LF extract did not provoke any changes on the structure of prepared NLS.

Two intense endothermic peaks were observed for both samples (Fig. 3D and E). The first peak was attributed to lecithin melting, and the second peak was associated with the melting of the sodium taurodeoxycolate counterpart (data not shown). Discreet endotherm peaks were also observed using a special zoom in the determined region of the DSC curve (Fig. 3E). These peaks were attributed to the melting point of the stearic acid.

The endothermic melting peak of soy lecithin was shifted to a higher temperature, from $174.5^{\circ} \mathrm{C}$ to $184.5^{\circ} \mathrm{C}$. Additionally, the absence of any degradation event of the LF extract in the LF-loaded SLN suggests that it is molecularly dispersed within the lipid matrix.

\subsection{Entrapment efficiency, drug loading and drug recovery}

The LF encapsulation efficiency was determined based on the quantification of YAN and EPI-YAN in the SLN suspension. Firstly, the method was fully validated demonstrating its applicability. Therefore, YAN and EPI-YAN were employed as markers, and the obtained results demonstrate the effectiveness of the markers in the formulation. The SLNs prepared in this study using the microemulsion technique showed high entrapment efficiency values for both markers, suggesting that the lipid and surfactant composition employed were suitable for the entrapment of markers in solid lipid nanoparticles. The obtained data are provided in Table 2.

The YAN and EPI-YAN encapsulation efficiencies were $94.21 \pm 0.40 \%$ and $94.20 \pm 0.00 \%$, respectively. These results 
A

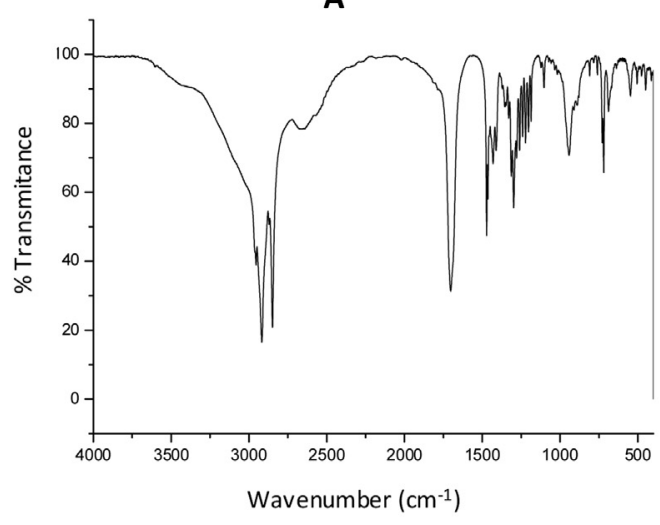

B

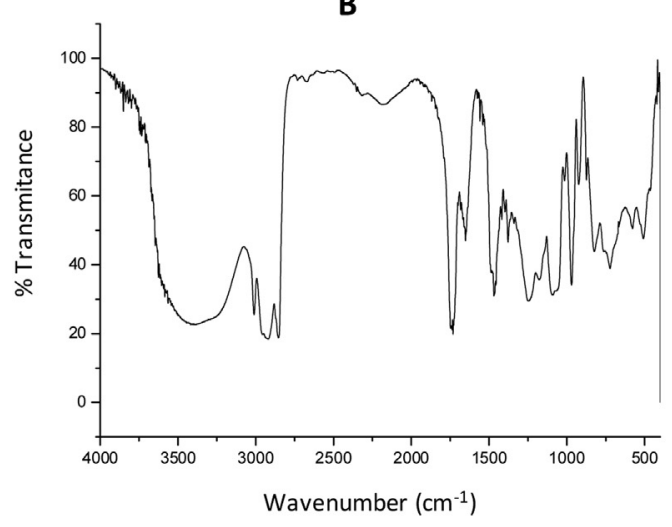

C

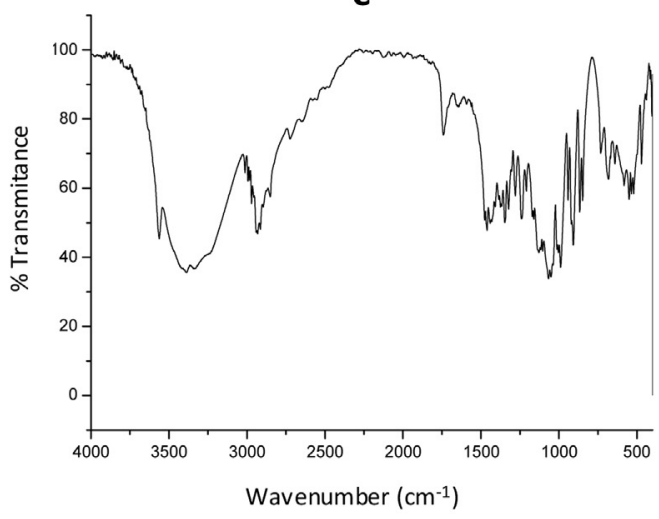

D

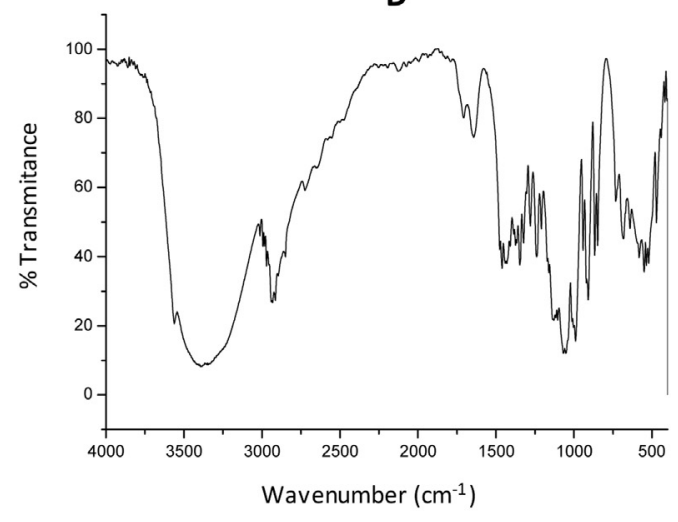

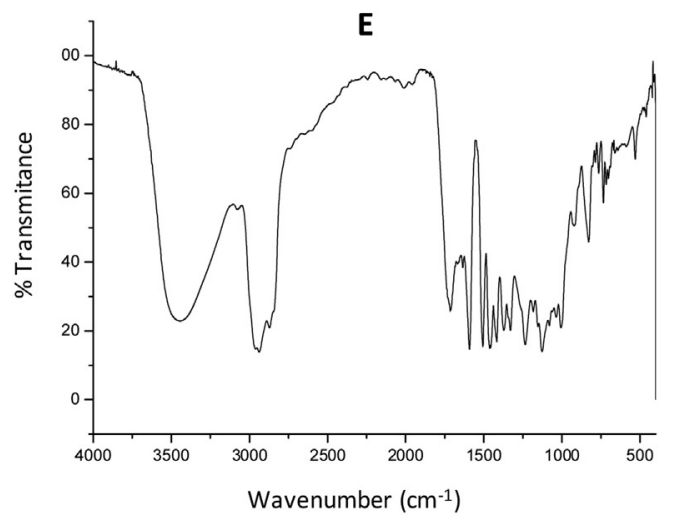

Fig. 2. Fourier Transformed Spectra for stearic acid (A), soy lecithin (B), unloaded-SLN (C), LF loaded-SLN (D) and LF (E).

Table 2

Entrapment efficiency, drug loading and drug recovery of LF-loaded SLN.

\begin{tabular}{|c|c|c|c|c|c|c|}
\hline Marker & $\begin{array}{l}\text { Nominal } \\
\text { concentration } \\
\left(\mathrm{mg} \mathrm{mL}^{-1}\right)\end{array}$ & $\begin{array}{l}\text { Obtained } \\
\text { concentration } \\
\left(\mathrm{mg} \mathrm{mL}^{-1}\right)\end{array}$ & Free drug $\left(\mathrm{mg} \mathrm{mL}^{-1}\right)$ & $\begin{array}{l}\text { Entrapment efficiency } \\
(\% \mathrm{w} / \mathrm{w})\end{array}$ & Drug loading $(\% \mathrm{w} / \mathrm{w})$ & Drug recovery $(\% \mathrm{w} / \mathrm{w})$ \\
\hline YAN & 0.398 & $0.242 \pm 0.007$ & $0.014 \pm 0.000$ & $94.21 \pm 0.40$ & $0.88 \pm 0.00$ & $60.77 \pm 1.99$ \\
\hline \multirow[t]{2}{*}{ EPI-YAN } & 0.500 & $0.295 \pm 0.010$ & $0.017 \pm 0.000$ & $94.20 \pm 0.00$ & $1.07 \pm 0.00$ & $59.21 \pm 2.08$ \\
\hline & $0.898^{\mathrm{a}}$ & $0.537^{a}$ & $0.031^{\mathrm{a}}$ & $94.21^{\mathrm{b}}$ & $1.95^{\mathrm{a}}$ & $59.80^{\mathrm{b}}$ \\
\hline
\end{tabular}

are higher than our previous work, in which these markers reached maximum EE\% 66.11 [15]. To date, the physical and chemical parameters of YAN or EPI-YAN have not been described in the literature, however, the high lipid solubility of these lignans has been observed by our group (data not shown). Three drug incorporation models are available for SLNs: (1) the solid solution model, (2) the core-shell model for a drug-enriched shell, and (3) the core-shell model for a drug-enriched core. melting point 
A

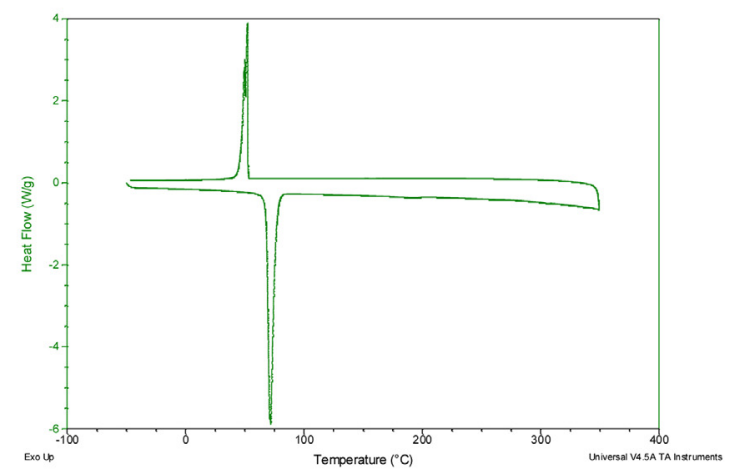

B

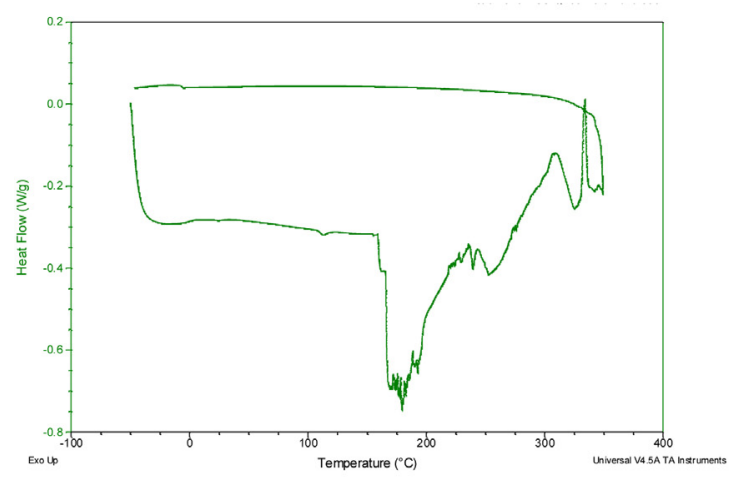

c

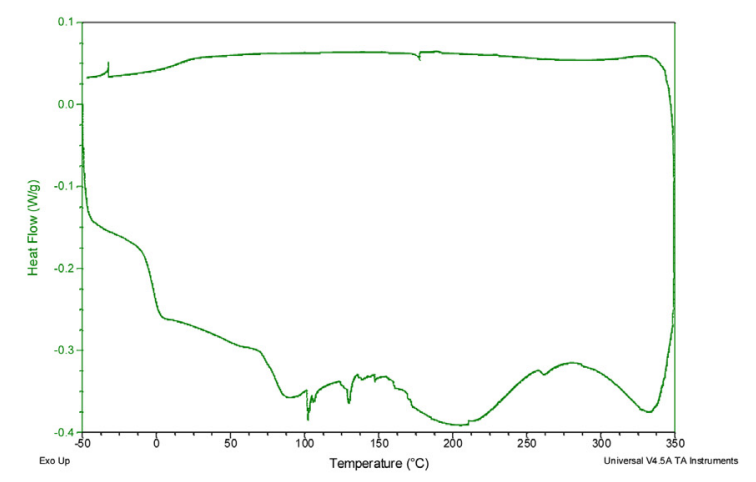

D

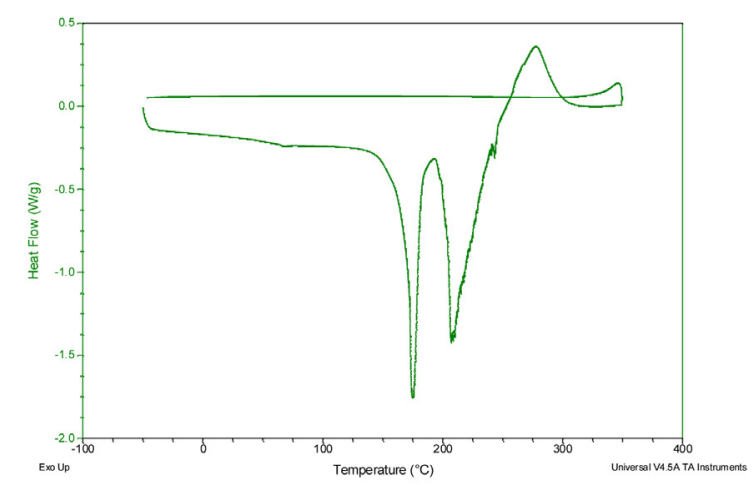

$\mathbf{E}$

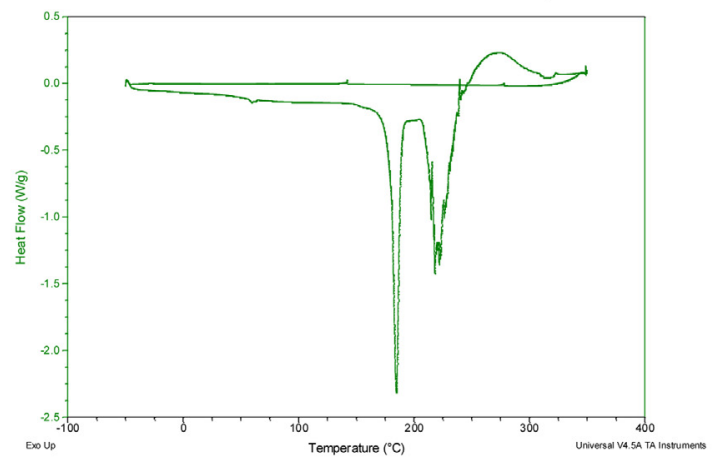

Fig. 3. Differential scanning calorimetric thermograms of (A) stearic acid, (B) soy lecithin, (C) Lignan Fraction (LF), (D) unloaded SLN and (E) loaded SLN.

differences between the lipids and drug agents may indicate the drug distribution in the SLNs [1]. Based on this discussion and the results of the characterization step, i.e., no changes in the zeta potential, FTIR and DSC data, the developed lipid carriers may follow the core-shell model (drug-enriched shell) of drug incorporation, which corroborates the release profiles of YAN and EPI-YAN from the lipid carriers (reported below). The drug-enriched core forms when the cooling of the hot oil/water emulsion precipitates the drug first. This phenomenon preferentially occurs in lipid solutions that are saturated with the drug at the lipid at production temperature, which facilitates supersaturation and subsequent precipitation during cooling [23].

The drug loading capacity is a very relevant parameter for judging the suitability of a particular drug-carrier system. In this study, the DL values reached $1.95 \%(\mathrm{w} / \mathrm{w}$ ) (see Table 2 ) when both markers were combined. However, this DL value was calculated only based on the amounts of markers. The estimated DL value for the entire lignan fraction was $9.0 \%$; this value is interesting because $1.83-5.51 \%$ loads of topotecan (a hydrophilic anti-neoplastic agent) [24] and 2.0-5.84\% loads of tamoxifen (a lipophilic anti-neoplastic agent) [25] have previously been reported. Moreover, although the loading of SLNs with natural extracts has been reported [26], the DL value has not yet been reported. This DL value may also be useful to produce drug preparations with acceptable weight and dimensions after the powder form of the suspension has been generated by lyophilization. In addition, data from the literature show that LF presents $\mathrm{IC}_{50}$ values for leishmania that are 20 - to 40 -fold lower than those of the reference drug [10], Glucantime ${ }^{\circledR}$ (meglumine antimoniate), which is marketed at a dose of $300 \mathrm{mg} \mathrm{mL}^{-1}$ i.v. Specifically, the reported $\mathrm{IC}_{50}$ values for $\mathrm{LF}$ are $26.5 \mu \mathrm{g} \mathrm{mL}-1$ against L. chagasi and $48.2 \mu \mathrm{g} \mathrm{mL}^{-1}$ against $L$. amazonensis, whereas these values exceed $1000 \mu \mathrm{g} \mathrm{mL}^{-1}$ for Glucantime ${ }^{\circledR}[12]$.

Regarding drug recovery (DR), the obtained value (approximately 60\%) is considered low, however, it could be mainly 


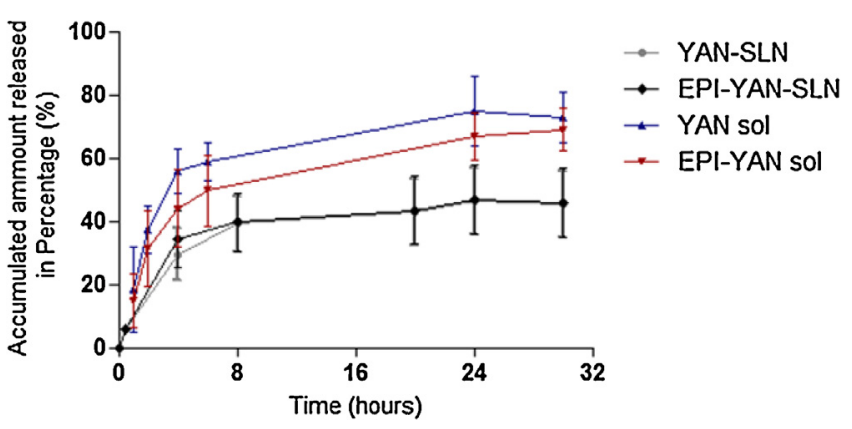

Fig. 4. In vitro YAN and EPI-YAN release profile in $\mathrm{pH} 7.4$ buffer for LF-loaded SLN, as well as LF alone (sink conditions attended). Data are given as means $\pm \operatorname{SD}(n=5)$.

attributed to the laboratorial scale. At this scale, the microemulsion, prior to SLN suspension obtention, is prepared in very small amounts, leading to important microemulsion mass loss in the laboratories utilities. Scaling-up will probably solve this problem. On the other hand, concerns regarding temperature must be taken into account, once it may also be responsible for low YAN and EPI recovery, due to possible degradations.

\subsection{In vitro kinetics of YAN and EPI-YAN release}

The YAN and EPI-YAN release profiles in buffer medium are presented in Fig. 4. Specifically $29.6 \pm 8.3 \%$ of YAN and $34.3 \pm 8.9 \%$ of EPI-YAN loaded in SLN diffuses through the cellulose acetate membrane (dialysis membrane) over $4 \mathrm{~h}$, reaching release rates of $45.5 \pm 10.5 \%$ for YAN and $45.9 \pm 11.0 \%$ for EPI-IAN after $24 \mathrm{~h}$. Moreover, both the YAN and EPI-YAN release rates from the lipid carrier are highly similar. Equilibrium was attained within $20 \mathrm{~h}$, which was confirmed by the plateau observed in the release profile between 20 and $30 \mathrm{~h}$. The differences in the release rates from SLN between these markers were not significant $(p>0.05)$ throughout the 20-30 h experiment.

The observed release pattern of the SLN could be due to the following sequence of events: the diffusion of free YAN and EPI-YAN during the first hour, diffusion from the solid lipid surface in the following hours and, subsequently, diffusion from the core. As indicated in Fig. 4, the YAN and EPI-YAN encapsulated within the core of the solid lipid are not completely released, even after $30 \mathrm{~h}$, i.e., the net drug release was only approximately $50 \%$. A quercetin-SLNs suspension reportedly exhibited sustained release and an accumulated drug release percentage of approximately $87.5 \%$ at $48 \mathrm{~h}$. The SLNs could reduce quercetin release in the latter stage, which was attributed to the fact that the solubilized or dispersed quercetin could only be slowly released from the lipid matrices via dissolution and diffusion [27]. This result was similar to those of many studies reporting that drug-loaded SLNs provided a controlled release pattern $[2,5]$. Both burst release as well as sustained release could be considered interesting for leishmaniasis treatment. Burst release is useful for improving the penetration of drug and accelerating the onset of action, whereas a sustained release supplies the drug over a prolonged period of time. On the other hand, the LF solution permitted to observe around $50 \%$ of each marker in 4-h study.

The drug release data from the LF-loaded SLN was fitted to different models (Table 3). The Higuchi model (pseudo first order model) yielded the highest $r$ value $(r=0.9950$ and 0.9945 for YAN and EPI-YAN, respectively), which indicates that the YAN and EPIYAN follow matrix diffusion-based release. After determining the model release, the diffusion coefficient of both markers $(D)$ was also determined. The diffusion coefficients were calculated using the Higuchi equation [28]. This parameter reflects the ease with which the molecules move through the formulation. The YAN and
EPI-YAN diffusion coefficient $(D)$ estimated after $8 \mathrm{~h}$ of experiment was $1.3 .10^{-6} \mathrm{~cm}^{2} / \mathrm{s}$ for both markers.

\subsection{Macrophage cytotoxicity assay}

Because the physical properties of the LF-loaded SLN nanoparticles were promising, the toxicity of the LF-loaded SLNs to mammalian macrophages was investigated, which are the preferred cells for infection by Leishmania. To this end, murine bone marrow-derived macrophages (BMDMs) were continuously treated with different concentrations of LF-loaded SLNs and unloaded SLNs $18 \mathrm{~h}$, and the cell viability was analyzed by quantifying membrane rupture via propidium iodide incorporation using a fluorometer device (Fig. 5). Specifically, intact membranes fail to internalize propidium iodide, whereas cells containing a pore or ruptured plasma membranes, as observed in dying cells, become permeable to this dye.

Previously, our research group verified that LF alone and its main constituents, YAN and EPI-YAN, were not cytotoxic to BMDMs, except when used at very high concentrations (equivalent to $80 \mu \mathrm{g} \mathrm{mL}^{-1}$ of LF fraction) [15]. At the present work, the same study was performed for unloaded SLNs or LF-loaded SLNs. Specifically, stimulating macrophages with 20 and $40 \mu \mathrm{g} \mathrm{mL}^{-1}$ unloaded SLNs or LF-loaded SLNs did not elicit cytotoxic effects, similar to the effect of free LF. But, interestingly, unloaded SLN or LF-loaded SLN remained nontoxic to macrophages at $80 \mu \mathrm{g} \mathrm{mL}^{-1}$, whereas free LF was toxic at this concentration (Fig. 5A-C). At first glance, these data suggest that the encapsulation of LF from Ocotea duckei Vattimo reduced the toxicity of this drug, but the amount of LF delivered to the cells per treatment period should be considered (approximately $40 \%$ of the compounds at 18 h, Fig. 4). Thus, the theoretical maximum concentration of $80 \mu \mathrm{g} \mathrm{mL}^{-1}$ is not available. Therefore, the safety of the high-dose LF-loaded SLN is not only related to the encapsulation effects but also to the decreased amount of LF available in the medium. Notably, macrophages were selected for this cytotoxicity assay, which are true immune cells. Thus, classical cell death, such as apoptosis or necrosis, and inflammatory cell death, known as pyroptosis can be evaluated [29]. Moreover, macrophages are the most suitable cells for studying new leishmanicidal drugs because the parasites establish and multiply within these cells.

\subsection{Anti-leishmanial activity of LF-loaded SLN}

After confirming the nontoxic and safe nature of LF-loaded SLNs, overcoming our previous results, their effect on the Leishmania parasite were assessed. To this end, Leishmania amazonensis was incubated with LF alone and LF-loaded SLNs for $24 \mathrm{~h}$ and their ability to kill the parasite was evaluated.

The optimum concentration of the LF fraction for antileishmanial action was based on previous studies. Monte-Neto and collaborators reported the anti-leishmanial activity of the crude ethanolic extract (CEE), lignan fraction (LF) and an isolated powder (called yangambin, containing both YAN and EPI-YAN as well as impurities) of Ocotea duckei. These authors observed that all three preparations exhibited high anti-leishmania activity. The lowest concentration of the LF fraction $\left(20 \mu \mathrm{g} \mathrm{mL}^{-1}\right)$ was significantly effective against both $L$. chagasi and L. amazonensis and presented an efficacy similar to that of Glucantime ${ }^{\circledR}$ at 500 and $1000 \mu \mathrm{g} \mathrm{mL}^{-1}$ [10]. Therefore, the $20 \mu \mathrm{g} \mathrm{mL}^{-1}$ of LF extract was assessed in the present study.

Fig. 6 shows the observed anti-leishmania activity of free LF alone and LF-loaded SLN. The leishmanicidal activity of LF-loaded SLN and free LF were similar. Nevertheless, the release rate of LF from the particles over $24 \mathrm{~h}$ (approximately 40-45\%) constitutes an improvement in the action of LF (Fig. 6). 
Table 3

Mathematical model of the drug release behavior of YAN and EPI-YAN present in LF-loaded SLNs.

\begin{tabular}{|c|c|c|c|c|}
\hline \multirow[t]{2}{*}{ Model } & \multicolumn{2}{|l|}{ YAN } & \multicolumn{2}{|l|}{ EPI-YAN } \\
\hline & Flux $(J)$ & $r$ & Flux $(J)$ & $r$ \\
\hline Zero-Order $\left(J=\mu \mathrm{g} / \mathrm{cm}^{2} \mathrm{~h}^{-1}\right)$ & $3.34 \pm 0,5$ & 0.9780 & $4.12 \pm 0.6$ & 0.9756 \\
\hline Pseudo First-Order $\left(J=\mu \mathrm{g} / \mathrm{cm}^{2} \cdot \sqrt{ } \mathrm{h}^{-1}\right)$ & $9.88 \pm 0.7$ & 0.9950 & $12.24 \pm 0.9$ & 0.9945 \\
\hline First-Order $\left(J=\log \mu \mathrm{g} / \mathrm{cm}^{2} \mathrm{~h}^{-1}\right)$ & $0.17 \pm 0.1$ & 0.8482 & $0.18 \pm 0.1$ & 0.8312 \\
\hline
\end{tabular}
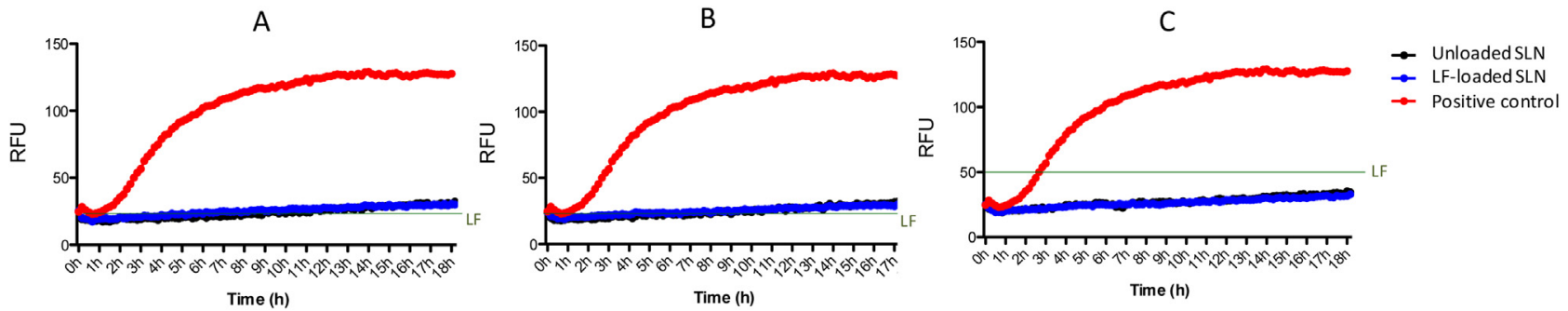

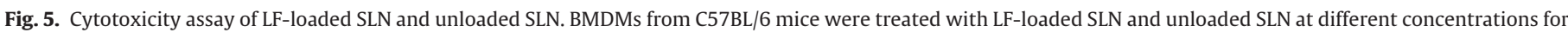

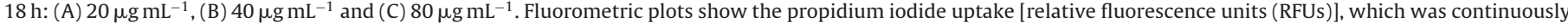

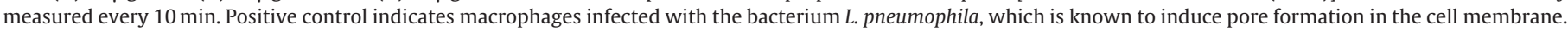

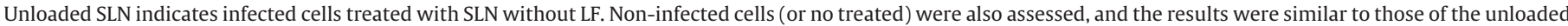

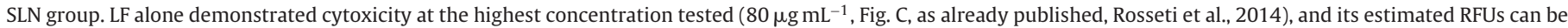
observed in this figure.

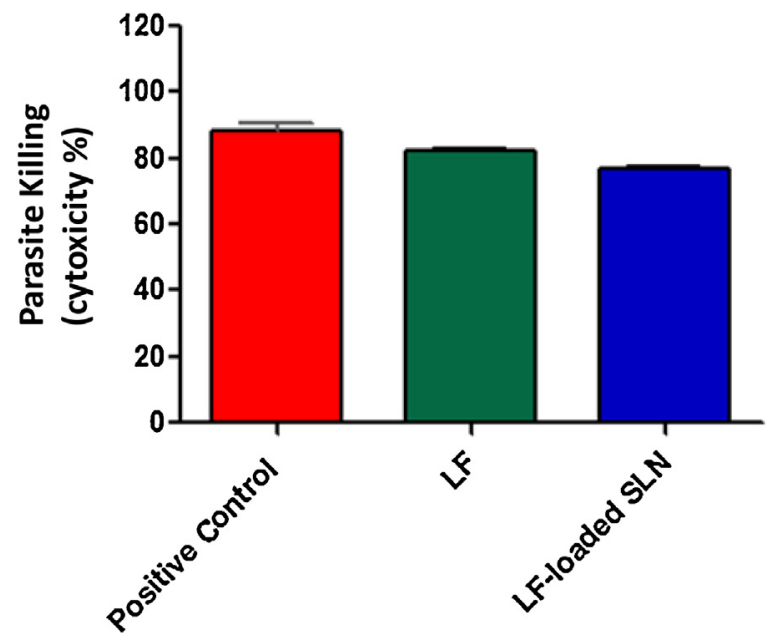

Fig. 6. Anti-leishmanial activity of LF-loaded SLN by MTT assay. Promastigote Leishmania amazonensis were seeded in 96-well plates at an initial density of $1 \times 105$ parasites/well and incubated with LF-loaded SLN and LF alone at $20 \mu \mathrm{g} \mathrm{mL}^{-1}$ for $24 \mathrm{~h}$ at $26^{\circ} \mathrm{C}$. The MTT was then, added and the resultant formazan was dissolved in SDS containing $0.01 \mathrm{M} \mathrm{HCl}$ after $4 \mathrm{~h}$. The absorbance was then measured at $570 \mathrm{~nm}$. Each column represents the mean \pm SD of at least three experiments. DMSO was employed as a positive control. No significant difference was observed among the studied groups (significance set to $P \leq 0.05$ in relation to pure LF, One-way ANOVA, followed by Dunn's Multiple Comparison Test).

In addition to increasing the selectivity, safety and efficacy LF against Leishmania, SLN carriers may also permit intracellular delivery to macrophages, as reported elsewhere. Although intracellular drug transport has been extensively studied, high target selectivity and therapeutic concentrations at the target subcellular compartments remain elusive due to intracellular complexity and dynamic conditions of nanoparticle release. Nevertheless, SLN have been shown to rapidly penetrate the keratinocyte membrane and move continuously through the cytoplasm after internalization to ultimately be delivered near the cell nucleus [14]. Via a combination of various experimental techniques, Kristl et al. have suggested the high dynamic trafficking of SLN in the cells and their localization in various subcellular compartments. SLN were not homogenously distributed in the cells but were localized predominantly between actin filaments and within mitochondria [30].

Overall, the data shown here demonstrate that the new carrier system presents safety and maintains or even improved the anti-leishmanial effect of Ocotea duckei Vattimo, which may be used to develop new plant-based drugs for Leishmaniasis treatment. Because Leishmania parasites survive intracellularly in host macrophages, the success of the encapsulation process of LF extracts and the promising results showed by LF-loaded SLN represent an important step in the development of a new treatment for this neglected disease.

\section{Conclusions}

The proposed LF-loaded lipid nanoparticle delivery system was demonstrated to be nanometer-sized with a zeta potential of $-30 \mathrm{mV}$. Furthermore, this system presents a high entrapment efficiency, as evidenced by a comprehensive physico-chemical assessment by FT-IR and DSC. The in vitro kinetics of YAN and EPIYAN release demonstrated that this delivery system follows the Higuchi model (pseudo first order model), indicating that YAN and EPI-YAN follow matrix diffusion-based release kinetics. Additionally, sustained drug-release was observed, as well as, LF-loaded SLN demonstrated to be safe for use in biological assays. Specifically, the cytotoxicity experiment indicated that these particles were nontoxic to mammalian macrophages, differently from our previous work. Finally, the lipid nanoparticle system improved the LF fraction effects because the anti-leishmanial effects of both loaded and unloaded LF were similar. However, only $40 \%$ of the loaded LF was released. Therefore, the LF-loaded SLN efficiently kills Leishmania, indicating that the lignan fraction of Ocotea duckei Vattimo associated with the solid nanoparticle delivery system is a promising new leishmanial drug.

\section{Conflict of interest}

The authors declare there is no conflict of interest. 


\section{Acknowledgments}

We would like to thank Prof. Dr. José Maria Barbosa Filho and Fabia Cristina Rossetti for the Ocotea duckei plant material, Professor Dra. Maria de Fátima Agra and Dra. Rita Baltazar de Lima for the identification of the plant material, Professor Dr. Dario S. Zamboni from FMRP-USP for the macrophage cytotoxicity assays, Apis Flora Indl. Coml. Ltda. for the facilities and the following Brazilian agencies for their financial support: CNPq, CAPES and FAPESP (Proc. nos. 2014/50410-3; 2011/51023-5).

\section{References}

[1] R.H. Müller, K. Mäder, S. Gohla, Solid lipid nanoparticles (SLN) for controlled drug delivery-a review of the state of the art, Eur. J. Pharm. Biopharm. 50 (2000) 161-177.

[2] Y. Wang, L. Zhu, Z. Dong, S. Xie, X. Chen, M. Lu, X. Wang, X. Li, W. Zhou, Preparation and stability study of norfloxacin-loaded solid lipid nanoparticle suspensions, Colloids Surf. B. 98 (2012) 105-111.

[3] J.G. Huang, T. Leshuk, F.X. Gu, Emerging nanomaterials for targeting subcellular organelles, Nano Today 6 (2011) 478-492.

[4] Z. Dong, S. Xie, L. Zhu, Y. Wang, X. Wang, W. Zhou, Preparation and in vitro, in vivo evaluations of norfloxacin-loaded solid lipid nanoparticles for oral delivery, Drug Deliv. 18 (2011) 441-450.

[5] S. Bose, B. Michniak-Kohn, Preparation and characterization of lipid based nanosystems for topical delivery of quercetin, Eur. J. Pharm. Sci. 48 (2013) $442-452$

[6] S.F. Taveira, D.C.A.S. de Santana, L.M.P.C. Araújo, F. Marquele-Oliveira, A. Nomizo, R.F.V. Lopez, Effect of iontophoresis on topical delivery of doxorubicin-loaded solid lipid nanoparticles, J. Biomed. Nanotechnol. 10 (2014) 1382-1390.

[7] F. Marquele-Oliveira, D.C.A. Santana, S.F. Taveira, D.M. Vermeulen, A.R.M. de Oliveira, R.S. da Silva, R.F.V. Lopez, Development of nitrosyl ruthenium complex loaded lipid carriers for topical administration: improvement in skin stability and nitric oxide release by visible light irradiation, J. Pharm. Biom. Anal. 53 (2010) 843-851.

[8] L.M. Negi, M. Jaggi, S. Talegaonkar, Development of protocol for screening the formulation components and the assessment of common quality problems of nano-structured lipid carriers, Int. J. Pharm. 461 (2014) 403-410.

[9] Leishmaniosis-World Health Organization (WHO). <http://www.who.int/ leishmaniasis/en/>(accessed 04.02.2014).

[10] R.L. Monte-Neto, J.M. Barbosa Filho, L.M.A. Sousa, P.F. Athayde-Filho, C.S. Dias, M.R. Oliveira, Crude ethanolic extract, lignoids fraction and yangambin from Ocotea duckei (Lauraceae) show antileishmanial activity, Z. Naturforsch., C, J. Biosci. 62 (2007) 348-352.

[11] R.L. Monte Neto, L.M.A. Sousa, C.S. Dias, J.M. Barbosa Filho, M.R. Oliveira, R.C.B.Q. Figueiredo, Morphological and physiological changes in Leishmania promastigotes induced by yangambin a lignan obtained from Ocotea duckei, Exp. Parasitol. 127 (2011) 215-221.

[12] R.L. Monte Neto, L.M.A. Sousa, C.S. Dias, J.M. Barbosa Filho, M.R. Oliveira, Yangambin cytotoxicity: a pharmacologically active lignan obtained from Ocotea duckei Vattimo (Lauraceae). Z. Naturforsch, C: J. Biosci. 63 (2008) 681-686.

[13] T.G. Ribeiro, M.A. Chávez-Fumagalli, D.G. Valadares, J.R. França, L.B. Rodrigues, M.C. Duarte, P.S. Lage, P.H.R. Andrade, D.P. Lage, L.V. Arruda, D.R. Abánades,
L.E. Costa, V.T. Martins, C.A.P. Tavares, R.O. Castilho, E.A.F. Coelho, A.A.G. Faraco, Novel targeting using nanoparticles: an approach to the development of an effective antileishmanial drug delivery system, Int. J. Nanomed. 9 (2014) 877-890.

[14] S. Martins, S. Costa-Lima, T. Carneiro, A. Cordeiro-da-Silva, E.B. Souto, D.C Ferreira, Solid lipid nanoparticles as intracellular drug transporters: an investigation of the uptake mechanism and pathway, Int. J. Pharm. 430 (2012) $216-222$.

[15] F.C. Rossetti, S.S. Leal, J.M. Barbosa Filho, E.J. Oliveira, H.S. Barud, J.I. Hori, F. Marquele-Oliveira, A.A. Berretta, Challenges in developing a safe nanomedicine based on Ocotea duckei Vattimo to leishmaniasis treatment: methodology, nanoparticle development and cytotoxicity assays, Pharm. Nanotechnol. 2 (2014) 101-114.

[16] F.M. Marim, T.N. Silveira, D.S. Lima Jr., D.S. Zamboni, A method for generation of bone marrow-derived macrophages from cryopreserved mouse bone marrow cells, PLoS One 5 (2010) e15263.

[17] C.L. Case, L.J. Kohler, J.B. Lima, T. Strowig, M.R. Zoete, R.A. Flavell, D.S. Zamboni, C.R. Roy, Caspase-11 stimulates rapid flagellin-independent pyroptosis in response to Legionella pneumophila, Proc. Natl. Acad. Sci. U. S. A. 110 (2013) 1851-1856.

[18] T. Mosmann, Rapid colorimetric assay for cellular growth and survival: application to proliferation and cytotoxicity assays, J. Immunol. Methods 65 (1983) 55-63.

[19] Z.A. Carneiro, P.I. da, S. Maia, R. Sesti-Costa, C.D. Lopes, T.A. Pereira, C.M. Milanezi, M.A.P. da Silva, R.F.V. Lopez, J.S. Silva, V.M. Deflon, In vitro and in vivo trypanocidal activity of H2bdtc-loaded solid lipid nanoparticles, PLoS Neglected Trop. Dis. 8 (2014) e2847.

[20] S.A. Saupe, A. Wissing, C. Lenk, R.H. Schmidt, Müller, Solid lipid nanoparticles (SLN) and nanoestructured lipid carriers (NLC)-structural investigations on two different carriers systems, Bio-Med. Mater. Eng. 15 (2005) 393-402.

[21 Z. Akbari, M. Amanlou, J. Karimi-Sabet, A. Golestani, M.S. Niassar, Preparation and characterization of solid lipid nanoparticles through rapid expansion of supercritical solution, Int. J. Pharm. Sci. Res. 5 (2014) 1693-1704.

[22] N. Choudhary, D. Pardhi, M. Bhoyar, Isolation of soy lecithin from soy sludge, its standardization and behavioural study, Asian J. Pharm. Clin. Res. 6 (2013) 133-136.

[23] M. Muchow, P. Maincent, R.H. Müller, Lipid nanoparticles with a solid matrix $\left(S N^{\circledR}, N^{\circledR} C^{\circledR}\right.$ LDC $^{\circledR}$ ) for oral drug delivery, Drug Dev. Ind. Pharm. 34 (2008) 1394-1405.

[24] L.G. Souza, E.J. Silva, A.L.L. Martins, M.F. Mota, R.C. Braga, E.M. Lima, M.C. Valadares, S.F. Taveira, R.N. Marreto, Development of topotecan loaded lipid nanoparticles for chemical stabilization and prolonged release, Eur. J. Pharm. Biopharm. 79 (2011) 189-196.

[25] C.W. How, A. Rasedee, S. Manickam, R. Rosli, Tamoxifen-loaded nanostructured lipid carrier as a drug delivery system: characterization, stability assessment and cytotoxicity, Colloids Surf. B. 112 (2013) 393-399.

[26] D.F. Cortés-Rojas, C.R.F. Souza, W.P. Oliveira, Encapsulation of eugenol rich clove extract in solid lipid carriers, J. Food Eng. 127 (2014) 34-42.

[27] H. Li, X. Zhao, Y. Ma, G. Zhai, L. Li, H. Lou, Enhancement of gastrointestinal absorption of quercetin by solid lipid nanoparticles, J. Control Release 133 (2009) 238-244

[28] W.I. Higuchi, Analysis of data on the medicament release from ointments, J. Pharm. Sci. 51 (1952) 802-804.

[29] B.T. Cookson, M.A. Brennan, Pro-inflammatory programmed cell death, Trends Microbiol. 9 (2001) 113-114.

[30] J. Kristl, K.T. Plajnšek, M.E. Kreft, B. Jankovicí, P. Kocbek, Intracellular trafficking of solid lipid nanoparticles and their distribution between cells through tunneling nanotubes, Eur. J. Pharm. Sci. 50 (2013) 139-148. 